P\&A Año 2, N.2

enero-junio de 2017

pp. $57-76$

Resumen

La fundación del Taller Integral de Diseño 8 a inicios del siglo XXI se produjo en un contexto de transición metodológica, caracterizado por la incipiente penetración de operadores tecnológicos en las aulas de diseño de la Facultad de Arquitectura y Urbanismo de la Universidad Ricardo Palma. Tras casi dos décadas de praxis y experimentación pedagógica, su posicionamiento como el primer taller virtual del Perú ha sido renovado gracias a la integración de los sistemas informáticos en todos los ámbitos de la actividad proyectual. Este trabajo revisa los valores fundacionales del taller como organización pedagógica; explora su nacimiento y evolución; y plantea una propuesta basada en su restructuración conceptual como un colaboratorio de innovación arquitectónica.

Palabras clave: Colaboratorio, taller de diseño, Arquitectura, sociedades del conocimiento

\title{
La emergencia del Taller Virtual como Colaboratorio de Innovación Arquitectónica*
}

\author{
The emergence of the Virtual Design Studio as an Architectural Innovation \\ Collaboratory
}

Bach. Arq. Gonzalo R. Chong Pascual**

Recibido: 10 de abril de 2017

Aceptado: 05 de junio de 2017

\begin{abstract}
The creation of the Integral Design Studio 8, in the beginning of the 21st century, occurred in a context of methodological transition, characterized by the incipient penetration of technological operators in the design classrooms of the Faculty of Architecture of Ricardo Palma's University. After two decades of praxis and pedagogical experimentation, its position as the first Virtual Design Studio of Peru has been renewed thanks to the integration of computer systems within all areas of architectural activity. This paper reviews the foundational values of the Virtual Design Studio as a pedagogical organization. It explores its birth and evolution, and presents a proposal based on its conceptual restructuration as an Architectural Innovation Collaboratory.
\end{abstract}

Keywords: Collaboratory, design studio, Architecture, knowledge societies

\footnotetext{
* El presente artículo es el resultado de las inquietudes y reflexiones del autor respecto a la evolución del Taller Virtual. Tras cerrarse un ciclo de dieciocho años bajo la jefatura del Arq. Félix Mayorca Palomino, y ante su sensible fallecimiento en el presente año, se examina la dinámica metodológica establecida por la cátedra y las posibilidades que esta ofrece como organización pedagógica. Es una investigación autofinanciada. Se declara no tener conflicto de intereses en la publicación de este artículo.

** Bachiller en Arquitectura y Urbanismo por la Universidad Ricardo Palma. Asistente de cátedra en el Taller Integral de Diseño Arquitectónico 8. Participó como asistente en la cátedra de Planeamiento de la Vivienda dirigida por la Arq. Doraliza Olivera Mendoza
} 


\section{In memoriam Arq. Félix Mayorca Palomino}

(1950-2017)

Nuestro taller, originalmente creado hace 10 años, tiene por finalidad cambiar el paradigma tradicional de la enseñanza de la arquitectura. En el año 1999 la forma tradicional de enseñar la arquitectura era a través de planos y maquetas. Nosotros, a propósito de los desarrollos de la informática, insistimos en crear una nueva forma, apoyándonos intensivamente en los medios de comunicación, y los avances tecnológicos sobre la computación. Durante estos diez años hemos desarrollado una experiencia que nos ha conducido por muchos caminos, todos ellos interesantes y seguimos buscando nuevos caminos, a la par del desarrollo de la tecnología, nuestro método ha ido también evolucionando (Mayorca, 2010a).

Al finalizar el siglo XX, emergió un panorama de transición metodológica afincado en la integración de nuevas tecnologías de diseño asistido por computadora (CAD por sus siglas en inglés) en las facultades y oficinas de diseño. Esto abrió el camino hacia una revisión del rol de la tecnología en la enseñanzaaprendizaje de la arquitectura dentro del contexto académico peruano. El Taller Integral de Diseño Arquitectónico N. ${ }^{\circ} 8$, gestado por iniciativa de los arquitectos ${ }^{1}$ Félix Mayorca Palomino, Walter León Távara y Carlos Huanambal Coral (ver Figura 1), fue inaugurado como sistema educativo en el año 1999. Surgió como un proyecto de innovación pedagógica que, desde la praxis educativa, proporcionó un espacio de búsqueda y experimentación abierto a una comunidad estudiantil en sintonía con un acelerado horizonte de desarrollo tecnológico. La cátedra, establecida en los laboratorios de cómputo de la Facultad de Arquitectura y Urbanismo (FAU) de la Universidad Ricardo Palma (URP), abrió sus puertas en el año 2000 como el primer taller virtual del Perú. Así, asumió el compromiso de aportar nuevas metodologías de trabajo y producción basadas en el aprovechamiento

1 En el mes de mayo de 2017, la cátedra sufrió la pérdida del Arq. Félix Mayorca Palomino, jefe y uno de los fundadores del Taller Virtual. De los tres arquitectos fundadores, permanece como parte del equipo de profesores el Arq. Walter León Távara. de las ventajas competitivas generadas por la creciente penetración de los sistemas informáticos en los campos asociados al diseño, la gestión de proyectos y la construcción. Tras 18 años de iteraciones metodológicas fundadas en una filosofía basada en el mejoramiento de la calidad de la educación "dentro de un marco de equidad, responsabilidad social y desarrollo humano y, sobre la base del desarrollo de procesos permanentes de innovación curricular" (Mayorca, 2010b, párr. 1), es meritorio plantear una revisión de los procesos fundacionales del Taller Virtual, a fin de evaluar su rol y proyección futura como parte integral y viva de la comunidad universitaria. Dicha reflexión parte de una lectura del ecosistema académico actual, evalúa la pertinencia de los medios digitales dentro de las aulas de la facultad de arquitectura e identifica los beneficios que su vínculo con la pedagogía ha otorgado a dicho ámbito. Esto ha ocurrido gracias a la consolidación de las tecnologías de la información y comunicación (TIC) como herramientas de diseño arquitectónico, su posicionamiento como medio de comunicación hipertextual ${ }^{2}$ y su inminente emergencia como espacio virtual de colaboración. Con el proceso actual de normalización tecnológica en los talleres de diseño integral, el enfoque del Taller Virtual ${ }^{3}$ ha evolucionado más allá de las preconcepciones derivadas de su motto ${ }^{4}$ característico y ha reafirmado su identidad como espacio de innovación pedagógica mediante la aplicación de una metodología basada en la enseñanza por compe-

2 Un hipertexto es un "conjunto estructurado de textos, gráficos, etc., unidos entre sí por enlaces y conexiones lógicas" (RAE, 2014, p. 63). Como concepto, está asociado a la informática, y alude a un sistema que permite el acceso y transferencia interactiva de información de manera no secuencial, a partir de fragmentos de texto relacionados por medio de enlaces asociativos hacia otros documentos.

3 Actualmente -el ciclo académico 2017-I- el personal de profesores se encuentra conformado por los arquitectos Walter León Távara, Pedro Hurtado Valdez, Amelia Fuentes Rocha y Ruth Suica Delgado. El equipo de asistentes está conformado por los bachilleres Gonzalo Chong Pascual, Xiomara Bautista Castilla y Miguel Caldas Mundo.

4 Motto es un término de origen italiano que refiere a una palabra u oración corta elegida para encapsular las ideas que guían a un individuo, grupo u organización. 


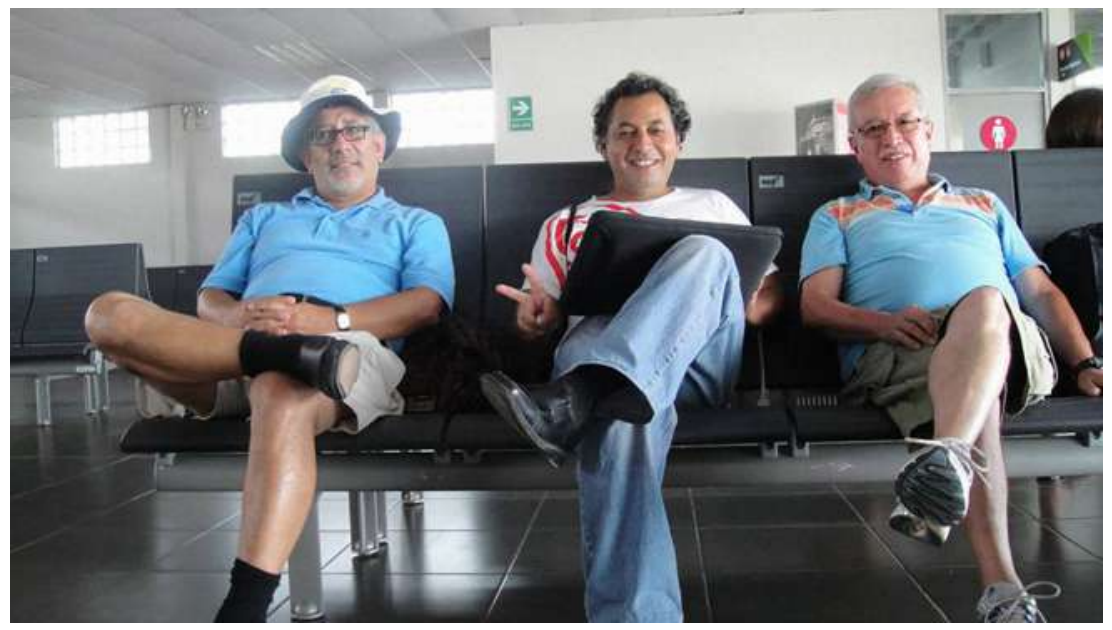

Figura 1. Los arquitectos fundadores del Taller Virtual. De izquierda a derecha: Arq. Walter León Távara, Arq. Carlos Huanambal Coral y Arq. Félix Mayorca Palomino [Fotografía por A. Fuentes Rocha, 2015, Lima, Perú].

tencias, la cual forma parte de los principios fundamentales del taller. El presente artículo tiene el propósito de estructurar una narrativa que expanda los alcances de este proceso sinérgico de construcción del conocimiento al retratar las inquietudes pedagógicas de la cátedra por encontrar un balance entre la conservación de los valores fundacionales del taller, así como la necesidad de producir un ambiente fértil para la innovación colaborativa. Para ello se realizará una revisión de los planteamientos del Taller Virtual a partir de la deconstrucción del taller tradicional de diseño, así como su evolución hacia un enfoque basado en la implementación de una cultura orgánica de aprendizaje colectivo. Finalmente, se explicarán, a modo de aporte, las posibilidades que ofrece la restructuración conceptual de la cátedra como un colaboratorio de innovación arquitectónica y se tomará como base el marco conceptual definido por Koichiro Matsuura (2005).

\section{La desfordización de la cátedra de Diseño Arquitectónico}

A diferencia de las materias presentes en la malla curricular de la Facultad de Arquitectura y Urbanismo, los talleres de diseño po- seen atribuciones complejas, fundadas en el rol pedagógico que cumplen como espacios de aprendizaje proyectual. En el contexto de la URP, su presencia curricular a lo largo de toda la carrera ha generado vínculos identitarios en el imaginario de los estudiantes, quienes a partir de sus experiencias en las aulas reconocen una correlación entre las características cualitativas de los talleres de diseño y la evolución de sus destrezas académicas personales. Este vínculo entre cátedra, identidad y crecimiento académico ha contribuido con el establecimiento de los talleres no solo como cursos regulares de carrera, sino como organizaciones pedagógicas con una cultura empresarial ${ }^{5}$ propia. Esta última las identifica y les permite competir en un mercado interno con alternativas temáticas diversas, basadas en la promoción de estilos particulares de entender y enseñar la activi-

5 A diferencia de los cursos regulares de carrera, los cuales poseen un número limitado de niveles y de redundancia respecto a la cantidad de alternativas ofrecidas por materia, los talleres de diseño existen en un ecosistema pedagógico de alta redundancia y de permanencia perenne con penetración en todos los niveles educativos de la carrera, por lo que existe un ambiente propicio para la competencia entre cátedras. 
dad proyectual. Por consiguiente, siguiendo una marcada orientación empresarial, las cátedras de diseño son fundadas reproduciendo un modelo jerárquico y piramidal, que las organiza bajo la forma de estructuras antropocéntricas administradas desde un enfoque top-down ${ }^{6}$. En dicho formato la visión y la misión están definidas por el jefe de la cátedra, y se distribuyen a través de un régimen en el que los profesores asumen las funciones de órganos locales de control y los alumnos o participantes las de los ejecutores de las directivas establecidas por los niveles superiores de la pirámide. Estos roles no son escalables ni intercambiables, de modo que cada subsistema jerárquico permanece circunscrito a su propio dominio de estabilidad funcional. Este esquema a la larga propicia la aparición de trampas de rigidez que reducen la resiliencia de la organización pedagógica ante la ocurrencia de disrupciones de origen administrativo ${ }^{7}$. La consolidación de estos patrones conductuales prefiguran la existencia de una cultura organizacional imbuida en un contexto de naturaleza equilibrada ${ }^{8}$, la cual, aplicada al ámbito pedagógico, puede ser descrita como un paradigma que vincula la supervivencia del taller de arquitectura a su capacidad para conservar su cosmovisión y estructuras orgánicas en el tiempo. En consecuencia, el surgimiento de modelos de pensamiento ajenos al núcleo identitario del taller puede producir rechazo al configurar perturbaciones sistémicas que fuerzan a la organización pedagógica a examinarse lejos de su región de estabilidad particular. Fuera

6 El enfoque top-down se estructura a partir de la existencia de una pirámide jerárquica en la que los niveles más altos implementan políticas con objetivos claros y consistentes, de modo que los niveles inferiores acatan dichas disposiciones, respetando el ejercicio de la autoridad vertical.

7 Administrativamente, los talleres existen en un entorno en el que la movilidad entre dominios funcionales se encuentra restringida por políticas externas a la organización, por lo que son entidades pedagógicas frágiles ante el impacto eventual de una disrupción en la continuidad funcional de la jefatura del taller, la cual por diseño es irremplazable e inaccesible por sucesión meritocrática desde el interior de la organización.

8 Según Gunderson y Holling (2002), la visión de naturaleza equilibrada existe en o cercana a un estado estable de equilibrio, y al ser perturbada recupera su estabilidad mediante procesos de retroalimentación negativa. de esta, pierde su integridad como producto educativo, dado que la apertura hacia procesos estructurales de innovación plantea a la larga la posibilidad de generar cambios de fondo que afectarían la coherencia entre la identidad, rol y función de la cátedra. En este contexto, los principios de diseño impartidos por los talleres de arquitectura, además de cumplir una función pedagógica, quedan instalados en la práctica como políticas de control diseñadas para perpetuar los valores identitarios de la organización. Asimismo, quedan establecidos, en palabras de Thomas Khun, como ciencia normal', validados a partir de su instalación en una masa crítica de estudiantes que alinean sus patrones de diseño a los códigos estilísticos de un taller particular y asumen de manera colateral la identidad del taller en el que participan. A partir de este esquema organizacional, los talleres de diseño ejercen un enfoque fordista ${ }^{10}$ del aprendizaje que opera como una línea de montaje en la que los alumnos son normalizados según los requerimientos de un programa pedagógico definido por la jefatura del taller. Dicho programa persigue la consecución de un ramillete de competencias específicas para cada nivel educativo, pero abordadas de manera distinta dependiendo del ideario particular de cada organización. De este modo, los alumnos se vuelven los receptores de un discurso arquitectónico predeterminado por los niveles superiores y que se ve reflejado en una producción académica también normalizada, como sinónimo de buen hacer proyectual. Con la sistematización de este enfoque se ha producido la parcelación de la cátedra de diseño en un conjunto coral de subsistemas temáticos. Estos están inherentemente integrados a una retórica que justifica la descomposición ${ }^{11}$ del proceso de creación pro-

\footnotetext{
9 Según Thomas Kuhn (1970), ciencia normal es la investigación basada en uno o más logros académicos del pasado, que la comunidad académica reconoce por un tiempo como base para su práctica profesional posterior.

10 Fordismo es un término derivado del nombre de Henry Ford. Este enfoque promueve la especialización, el uso de esquemas industriales y la reducción de costos. 11 El verbo descomponer es utilizado según la segunda acepción que figura en la web del Diccionario de la Real Academia Española: "Separar las diversas partes que forman un compuesto" (2014, párr. 2).
} 
yectual, e instalan una visión reduccionista de la arquitectura como un objeto desarticulable y de su aprendizaje como un proceso de ingeniería inversa ${ }^{12}$, aplicado al estudio de tipologías arquitectónicas predefinidas por el taller de diseño.

Como organización pedagógica, el Taller Virtual fue fundado de manera tradicional, adoptando una estructura jerárquica piramidal, la cual se mantuvo bajo la jefatura del Arq. Félix Mayorca Palomino durante 18 años, hasta el final de su ciclo vital el mes de abril del presente año. Sin embargo, al haber nacido como un proyecto educativo impulsado gracias a una iniciativa grupal, su evolución se separó de los patrones fordistas habituales y siguió un principio organizacional claro: la cátedra de diseño no es un sistema cerrado y monolítico que opera en un paisaje estático de estabilidad jerárquica, sino que, por el contrario, se desenvuelve en un mundo plano que permite una distribución horizontal de la toma de decisiones a través de la práctica de procesos de gobierno colectivo. Por consiguiente, la dinámica organizacional del taller se desarrolló a través del establecimiento de una estructura panárquica ${ }^{13}$ fundada en las relaciones de interdependencia entre los niveles permanentes, los cuales proporcionan las directivas generales que dan orientación y propósito al taller de manera consensuada; y los niveles efímeros, conformados por los participantes del curso, quienes representan la fuerza creativa del sistema al constituirse como motores de innovación y producción de nuevos paradigmas arquitectónicos. Este esquema elimina la separación tradicional entre cátedra y alumnado, y los integra como parte de una sola organización pedagógica,

\footnotetext{
12 El término ingeniería inversa es utilizado para hacer referencia al proceso de descubrir los principios que rigen la creación de un objeto o pieza de ingeniería, analizándolo a partir del estudio de sus piezas constitutivas y utilizar el pensamiento abductivo con el fin de crear una reproducción del mismo.

13 La panarquía es una forma de organización propia de los sistemas complejos adaptativos que rompe con las nociones tradicionales de jerarquía, al establecer un funcionamiento basado en la consecución de ciclos de prosperidad, declive e innovación. Esto permite la evolución del sistema tras adaptarse y transformarse ante la aparición impredecible de cambios en el contexto o de factores de perturbación externa.
}

a través del uso de la gestión participativa como medio para balancear los procesos de conservación gestionados por los subsistemas administrativos, los cuales están encargados a los mentores; y de innovación, movilizados por los subsistemas productivos a cargo de los alumnos. Además, establece desde la praxis la noción de que todos y cada uno de los subsistemas que integran el Taller Virtual pueden aportar bloques vitales de conocimiento dentro de las diferentes escalas o niveles del sistema (ver Figura 2). Con esta base, la cátedra ha funcionado aplicando una enfoque bottom-up $p^{14}$ basado en el uso de estrategias sinérgicas de aprendizaje participativo, de modo que "el objeto central es el diseño urbano-arquitectónico en sus múltiples tipologías y escalas morfológicas entendiendo que el estudiante es un constructor activo y el docente un facilitador del aprendizaje" (Mayorca, 2010b, párr. 1). Este proceso de flexibilización jerárquica permitió la apertura de la cátedra hacia un contexto pedagógico de naturaleza evolutiva ${ }^{15}$, en el que la sostenibilidad del proyecto educativo no es medida solo por su capacidad para persistir, sino también para evolucionar y reinventarse de forma permanente. Desde esta visión, el Taller Virtual asumió como núcleo identitario el abordaje de la crisis instrumental generada por el impacto de los sistemas informáticos en la actividad proyectual, el cual, al estar pautado por la velocidad incremental del desarrollo tecnológico, propició procesos permanentes de innovación curricular encaminados a "replantear todo el andamiaje sobre las escasas y nuevas formas de la enseñanza de la arquitectura en nuestro país" (Mayorca, 2010b, párr. 1). Para ello, la cátedra erigió un estilo pedagógico orientado al aprendizaje

\footnotetext{
14 El enfoque bottom-up surge como crítica a las políticas top-down, y analiza la relación entre los burócratas y a quienes están dirigidas sus políticas. Así, el proceso de implementación se desarrolla a través de una red de actores más que a través de una estructura administrativa rígida y jerárquica.

15 Según Gunderson y Holling (2002), la visión de naturaleza evolutiva es un paradigma de cambio abrupto y transformador que expone la necesidad de comprender la dinámica impredecible de los ecosistemas; describe un paisaje de estabilidad que cambia retroalimentativamente mediante la autoorganización.
} 


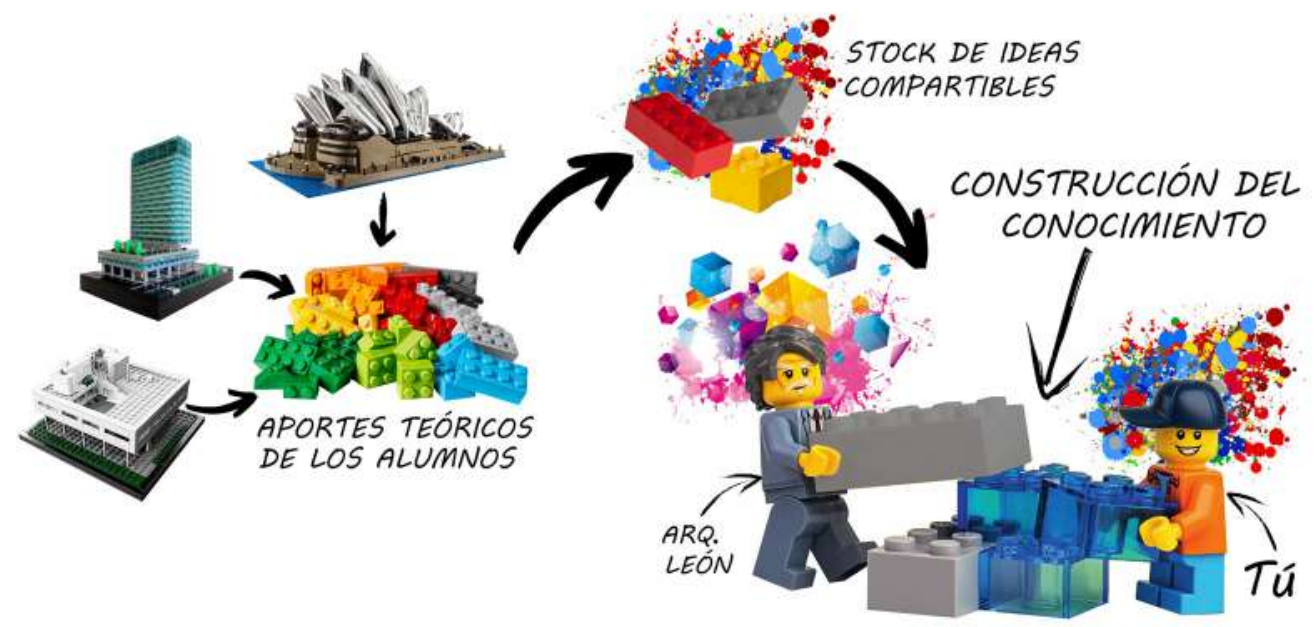

Figura 2. Enfoque teórico por medio del concepto de construcción del conocimiento. [Material gráfico de apoyo utilizado durante la apertura del ciclo 2017-I para exponer la filosofía del taller al alumnado, elaboración propia, 2017].

basado en problemas con el fin de que los temas a ser abordados en el taller, además de cumplir con los objetivos técnico-formativos del curso, establecieran lazos retroalimentativos con la problemática nacional, y generen soluciones vinculadas a un contexto global de avance tecnológico. Consecuentemente, el taller abordó una mirada "glocalizada"16 de la labor proyectual sostenida desde la implementación de procesos iterativos de innovación arquitectónica, pero coherentes con las particularidades del contexto nacional. Para lograr la implementación de este enfoque, el taller partió de una premisa central: los proyectos académicos no son elaboraciones intelectuales autocontenidas en una retórica puntual e inmanente, sino que surgen como formulaciones que trascienden los contornos plásticos del proyecto. De este modo, su calidad como objeto arquitectónico se encuentra vinculada paramétricamente a las variables

16 Glocalización es un término que reúne los conceptos de globalización y localización, el cual define la adaptación de productos, servicios y tecnologías internacionales a las particularidades de un contexto cultural local, con el fin de crear versiones ecualizadas compatibles con la realidad del lugar en la que son implementadas. presentes en el espacio-tiempo histórico en el que se desarrolla la cátedra, y da como resultado la producción de una arquitectura con enfoque sistémico, sostenible y resiliente. Esta concepción "socio-ecosistémica"17 de la labor proyectual se encuentra contenida en los principios del Taller Virtual (ver Figura 3), donde se establece que sus acciones se sustentan en el objetivo de "formar profesionales capaces de respetar el medio ambiente, el desarrollo sostenible, la investigación ambiental, la innovación tecnológica, el eco-sistema, la identidad nacional y la ética profesional, comprometidos con el bienestar común de la sociedad" (Mayorca, 2010b, párr. 10). Desde este modelo de pensamiento, los paradigmas arquitectónicos no constituyen dogmas, sino que poseen un carácter instrumental cuya construcción, adaptación o adopción persigue un propósito específico: fungir como el sistema operativo a través del cual los estudiantes

17 El atributo socio-ecosistémico hace referencia a la naturaleza de un sistema en los que los procesos sociales tienen un impacto fundamental en la evolución, adaptación y transformación de los sistemas ecológicos, dado que es la escala de la actividad humana la que modela el entorno físico y ambiental en el cual se desarrolla. 


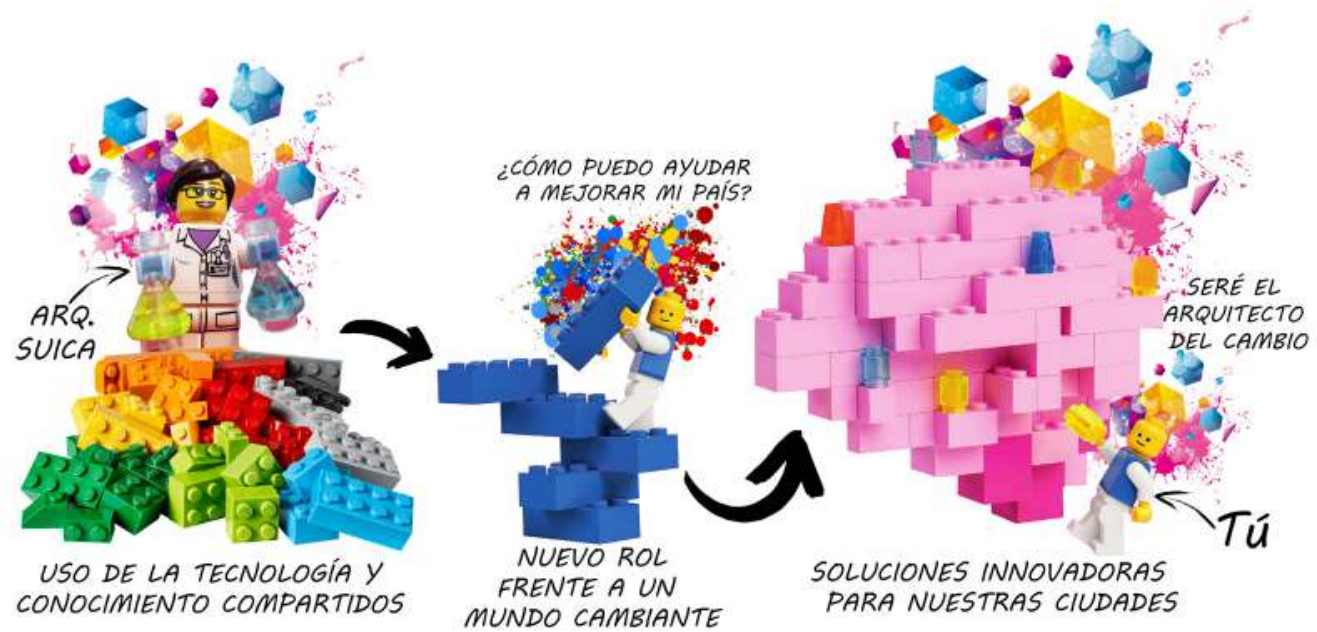

Figura 3. Enfoque socio-ecosistémico por medio del concepto de innovación disruptiva [Material gráfico de apoyo utilizado durante la apertura del ciclo 2017-I para exponer la filosofía del taller al alumnado, elaboración propia, 2017].

estructuran toda la información producida en la formulación sistémica del proyecto arquitectónico.

\section{La descosificación instrumental del proyecto arquitectónico}

En sus inicios, parte del trabajo fundacional del Taller Virtual consistió en vencer la resistencia al uso de operadores tecnológicos en las etapas de conceptualización y prototipado de los proyectos de diseño. Este escenario de inercia metodológica estuvo reforzado por la persistencia de una percepción reduccionista respecto al lugar del ordenador dentro del proceso creativo, cuyo uso, comúnmente acotado para fines de presentación arquitectónica, era considerado solo como un reemplazo del tablero de dibujo debido a su utilidad como instrumento digital de delineado y representación fotorrealista. Sin embargo, con la llegada del nuevo milenio, se obtuvo un argumento en contra de este enfoque superficial. Esto se debió, en parte, a la consolidación de nuevas formas de expresión posmodernistas provenientes de personalidades icónicas como Frank Gehry, Zaha Hadid,
Rem Koolhass, Herzog y De Meuron, así como de la mano una larga lista de arquitectos contemporáneos, quienes desde la práctica, en sus estudios particulares, contribuyeron a desmitificar el estigma de lo digital como elemento de desnaturalización del acto humano de creación arquitectónica. De hecho, los argumentos relacionados a las ventajas perceptuales y cognitivas de los modelos físicos, versus las ventajas técnicas asociadas a los modelos digitales, constituyen en sí mismos elementos de un retórica sesgada a partir de un enfoque netamente tecnocentrista del impacto de los medios informáticos sobre la labor proyectual ${ }^{18}$. Este desacuerdo se puede matizar si se analizan los procesos de trabajo

18 Un ejemplo del discurso que cuestiona el uso actual de las TIC en el proceso de aprendizaje de la arquitectura se puede encontrar en el artículo "Docentes Analógicos, Estudiantes Digitales", publicado por la revista Escala, donde se expresa que "por las facilidades que ofrecen a la hora de imaginar, los programas engañan y hacen creer al individuo promedio que realmente puede crear y concebir arte, o peor aún, afectan el verdadero talento de alguien que si lo pueda poseer. El software se ha convertido en instrumento fundamental que genera necesidades enfermizas y someten a un artista o a los futuros arquitectos a los conocimientos que posea del programa" (Sánchez, 2012, p. 126). 
de arquitectos de la talla de Eero Saarinen, quien en los años 50 ya había experimentado las limitaciones derivadas del uso de soportes tradicionales de representación arquitectónica aplicados en la producción de geometrías orgánicas de escala monumental, como las diseñadas para el Terminal de la TWA. El caso resulta emblemático, porque si bien la comunidad profesional ha elogiado el heroísmo de los métodos artesanales de Saarinen, basados en el uso de maquetas de gran esca$1 \mathrm{a}^{19}$, este en realidad optó por recurrir a las técnicas que el avance tecnológico de la época puso a su disposición para lograr el mismo objetivo que persigue actualmente la arquitectura digital: la construcción de nuevos paradigmas espaciales a través de la producción de simulaciones realistas del objeto arquitectónico proyectado. Medio siglo después, es a través de la construcción y popularización de obras como el Museo Guggenheim de Bilbao o el Estadio Nacional de Beijing, que se ha podido contar con un registro testimonial del amplio potencial de los sistemas computacionales. Estos no solo funcionan para generar simulaciones de alta calidad que permitan escudriñar los distintos componentes del proyecto meses antes de su construcción, sino también para gestionar la producción de envolventes e interiores con elevados niveles de complejidad geométrica-expresiva a escala tanto bidimensional como tridimensional, lo

\footnotetext{
19 Eero Saarinen fue conocido por recurrir en su estudio a la elaboración de maquetas de gran tamaño que le permitían introducirse físicamente en su interior, con el fin de obtener una percepción tridimensional e inmersiva de los espacios y envolventes proyectados. Sin embargo, cabe señalar que la elaboración y re-elaboración de este tipo de modelos, causados por modificaciones y decisiones de diseño posteriores, implicaron, por un lado, un consumo desmesurado de tiempo, que en el caso de la Terminal de la TWA resultó en la ampliación del plazo en un año para poder terminar el proyecto. Además, al margen de la incuestionable calidad arquitectónica del producto final, este enfoque planteó dificultades para generar una base planimétrica compartible con las distintas especialidades ingenieriles asociadas al diseño del terminal, dado que su traducción al lenguaje bidimensional debía realizarse trasladando las cotas extraídas directamente de las maquetas de trabajo hacia los planos arquitectónicos, configurando flujos de trabajo poco eficientes.
}

que permite su codificación ${ }^{20}$ en función de un amplio abanico de parámetros de orden plástico, energético, estructural, acústico, de confort, entre otros. En consonancia con este contexto de transición hacia un enfoque digital en la elaboración de proyectos, el Taller Virtual planteó una posición clara respecto a la pertinencia de integrar las TIC a la praxis pedagógica, no solo como elementos periféricos de enseñanza, sino también como insumo, herramienta y espacio de producción arquitectónica. Motivado por la exploración de un paisaje tecnológico en ciernes, el taller sostuvo, durante su etapa inicial, una visión purista respecto al uso de los sistemas informáticos como medios de expresión creativa, y procuró la inducción del alumnado hacia un proceso de desapego de los medios tradicionales de representación arquitectónica ${ }^{21}$ con el objetivo de facilitar su apertura hacia una nueva metodología digital que propiciase la construcción de un lenguaje conceptual propio (ver Figura 4). La emergencia de este lenguaje virtual ${ }^{22}$, liberado de los heurísticos ${ }^{23}$ inherentes a las propiedades físicas del mundo material, supuso un impacto cognitivo que, explorado desde la hipótesis de SapirWhorf ${ }^{24}$, influenció la manera en la que el alumnado se aproximó a la racionalización del proyecto arquitectónico, dado que los mismos comandos y condiciones espaciales

20 Se utiliza el término codificiación para aludir a la palabra anglosajona coding, la cual define la actividad de escribir líneas de código por parte de los profesionales dedicados el desarrollo de software. Este reemplaza al término programación, el cual ya posee un uso específico en el lexicón arquitectónico.

21 Este tipo de estrategias son utilizadas hoy en día por muchas oficinas de diseño, las cuales con el fin de realizar un upgrade tecnológico restringen el proceso de producción únicamente al uso del software a ser adoptado.

22 La palabra virtual indica, en esta frase, las características del argot desarrollado en el Taller Virtual, y une la terminología convencional con los términos provenientes del ámbito del software.

23 Los heurísticos son definidos en el campo congnitivo como atajos de la mente, o estrategias de solución de problemas condicionados por un conjunto de experiencias empíricas.

24 La hipótesis de Sapir-Whorf establece que existe una cierta relación entre las categorías gramaticales del lenguaje que una persona habla, y la forma en que la persona entiende y conceptualiza el mundo. 


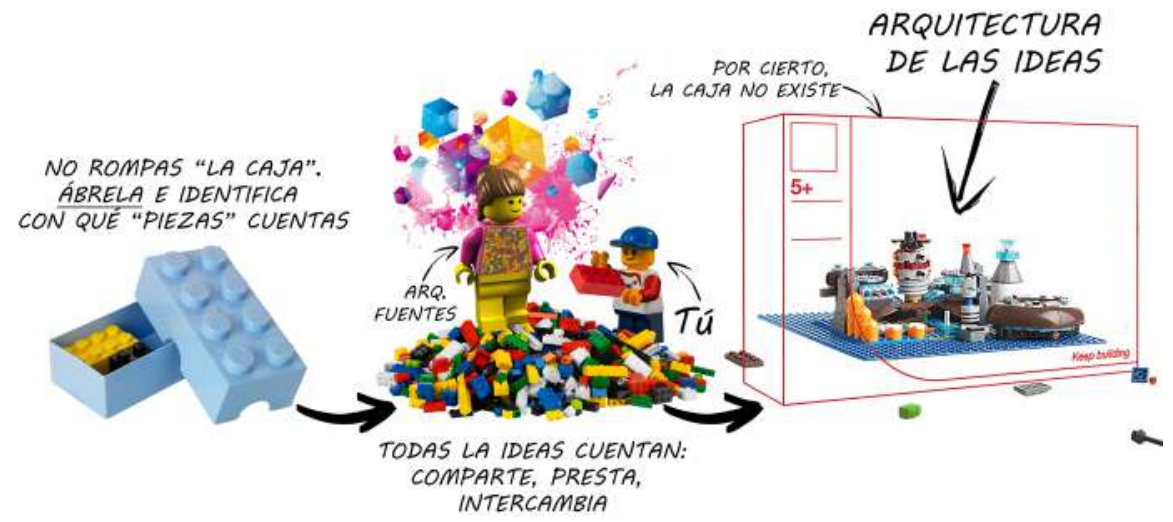

Figura 4. Virtualización del proceso de creación proyectual [Material gráfico de apoyo utilizado durante la apertura del ciclo 2017-I para exponer la filosofía del taller al alumnado, elaboración propia, 2017].

utilizados en los procesos de modelado a través de los softwares de diseño comenzaron a ser aplicados para expresar ideas abstractas relacionadas a la naturaleza de los prototipos de trabajo en desarrollo. En consecuencia, la sustancia de transferencia digital utilizada para representar las ideas de diseño instaló en el imaginario proyectual el reflejo de un mundo virtual, en el que las líneas, superficies y volúmenes poseen el potencial de ser alterados de maneras infinitas, y abrió nuevos grados de libertad creativa apoyados en el uso de elementos de composición inmateriales pero maleables desde la interfase virtual de un ordenador. Desde una perspectiva semántica, este proceso sinérgico de creación del lenguaje trajo consigo la adopción de un lexicón enriquecido a partir de términos y conceptos provenientes del ámbito digital, y configuró en su conjunto un dialecto empleado por los participantes de la cátedra, producto de las extensas sesiones de trabajo dedicadas a modelar $^{25}$ y renderizar ${ }^{26}$ sus prototipos de di-

25 Modelar es generar la representación matemática de un objeto tridimensional utilizando un softwares especializado.

26 Renderizar es un término proveniente del inglés render, y se refiere al acto de generar una imagen o video partiendo de un modelo 3D, y simular el comportamiento de la luz, materiales y propiedades físicas. seño. Operativamente, estas nuevas maneras de conceptualizar y expresar las ideas arquitectónicas generaron un período de descosificación instrumental al interior del taller mediante la homologación de las distintas instancias físicas-analógicas utilizadas en los talleres tradicionales, lo que las convirtió en instancias virtuales-digitales destinadas a codificar toda la densidad de datos producidos por los alumnos durante el aprendizaje de su actividad proyectual. De esta manera, entregables, como dibujos y esquemas conceptuales, fueron plasmados en presentaciones en formato PPT, lo que además explotó las características dinámicas de los programas de edición de imágenes y favoreció la construcción de un lenguaje visual más elaborado. Los documentos imprimibles fueron sustituidos por archivos de extensión CAD, los cuales podían ser revisados y medidos a un nivel de detalle más profundo que los planos físicos de escala estática, además de permitir sugerir cambios rápidamente actualizables a partir de comandos sencillos ejecutados desde el ordenador. Las maquetas de trabajo fueron reemplazadas por modelos virtuales en formato MAX, que no solo podían contener información a nivel de envolvente, sino también, mediante el uso de cámaras virtuales, podían permitir su libre inspección exterior e 
interior, simulando recorridos con diferentes condiciones de iluminación y acabados. Sin embargo, cabe subrayar que la fluidez de esta transición metodológica fue posible no solo por la eficiencia de los sistemas informáticos adoptados, sino también gracias a la implementación de una cultura abierta de colaboración entre profesores, asistentes y alumnos. Es esta etapa la que sentó las bases para la creación de un ambiente educativo particular que proporcionó a los estudiantes la oportunidad de explorar nuevos lenguajes emergentes, analizados, por un lado, desde la especulación plástica de la cosa arquitectónica ${ }^{27} \mathrm{y}$, por otro lado, desde una visión sistémica del proyecto mediante el uso de las herramientas de modelado digital como plataforma de innovación multimodal y de comunicación. Como corolario de este proceso de descosificación de la actividad proyectual, el Taller Virtual ha logrado hacer de la virtualidad de sus procesos pedagógicos su fortaleza y emblema identitario al interior de la comunidad académica, atributo que le ha permitido extender el alcance de su actividad formativa fuera de los límites físicos del aula de estudio, y ha consolidado su estatus de espacio de experimentación gracias a su integración a la red internacional del Taller Virtual de Las Américas $^{28}$ (TVA), el cual "como un sistema educativo interactivo de participación colaborativa de enseñanza y aprendizaje entre un conjunto de Universidades de diferentes países de América,[...]se extendió a muchas Escuelas de Arquitectura de este continente" (León, 2016, p. 1). Originalmente, universidades de Estados Unidos, México, Guatemala, Perú y Brasil se integraron al programa en el año 2002, y se sumaron a un conglomerado

27 Según Christian Norberg-Schulz (2008), "cuando una imagen arquitectónica aúna cualidades espaciales y plásticas, se convierte en una 'cosa arquitectónica' que forma parte de una obra arquitectónica" (p. 209).

28 En 2010, la Universidad Ricardo Palma, por medio de la participación de Mido Mahmoud Orejuela Chiriboga, ocupó el tercer lugar en los premios individuales del TVA. En el año 2012, ocupó el primer lugar con la propuesta de María Claudia Ángeles Velásquez. En 2013, Milenko Chlebowsky Uemura ocupó el segundo lugar en los premios individuales, y en 2015 sucedió lo mismo con Jesús Gonzalo Margarito Palacios (León, 2016). de más de 30 instituciones educativas participantes de una competencia internacional de diseño arquitectónico desarrollada íntegramente en el espacio virtual de la nube. Actualmente, la URP participa en los meses correspondientes al segundo semestre académico, y cuenta con el compromiso de los alumnos del noveno y décimo ciclo del Taller Virtual, quienes siguen un estricto cronograma fijado por el TVA y reciben, además, críticas de profesores y estudiantes de otras universidades vía correo electrónico o por medio de teleconferencias (ver Figura 5). Mediante el uso de blogs personales, los alumnos realizan la entrega de soportes digitales como infografías, planos, vistas 3D y videos, y las hacen accesibles para su libre escrutinio por parte de sus pares internacionales. Huelga decir que, en esta competencia, la herramienta primordial del Taller Virtual no son los cables y circuitos de silicio en el ordenador, sino el uso y dominio de un lenguaje proyectual construido como un sistema de comunicación de código abierto. Esta metodología ha proporcionado a los alumnos la oportunidad de utilizar la informática desde un enfoque que excede el tecnocentrismo reduccionista ejercido por los talleres de diseño tradicionales, y demuestra su potencial como puente de conexión semántica hacia un ambiente fértil de intercambio multicultural (ver Figura 6). Terminada la competencia, la cátedra examina los procesos y paradigmas espaciales generados por los estudiantes en el TVA, y los integra a un repertorio conceptual que es sintetizado y utilizado como insumo para renovar e "impartir competencias urbano-arquitectónicas coherentes con las leyes y principios de la percepción humana a través de estructuras espaciales dirigidas al confort habitacional mediante estímulos a experiencias sensoriales" (Mayorca, 2010b, párr. 11), y cierra así un ciclo iterativo de aprendizaje colaborativo.

\section{De la deslocalización a la ubicuidad del proceso pedagógico}

Tras cumplirse casi dos décadas de la aparición del Taller Virtual, la pertinencia de la virtualidad en el panorama pedagógico 